\title{
A GENERALIZATION OF THE EILENBERG-MOORE SPECTRAL SEQUENCE
}

\author{
BY D. W. ANDERSON
}

Communicated by E. H. Spanier, February 24, 1972

The purpose of this note is to announce a generalization of the (cobar) spectral sequence of Eilenberg and Moore. Full proofs and further applications will be given elsewhere.

In this paper, "space" will mean simplical set, though this is not essential to our arguments, and topological spaces could be used with some modifications.

Suppose that $X$ is a cosimplicial space. If $K$ is a field, let $K(X)$ be the cosimplicial simplicial vector space which is obtained by taking the degreewise free $K$-vector space on the sets $X[m](n)$ (the square brackets refer to cosimplicial degree, the parentheses to simplicial degree). There is a cosimplicial map $X \rightarrow K(X)$ which is the extension of the set map which sends each element into the basis vector which it determines. If || denotes the "geometric (co)realization" in the sense of Bousfield and Kan [1], there is a realization $|X| \rightarrow|K(X)|$ of this map. Since $|K(X)|$ is easily seen to be a $K$-vector space, we see that there is an obvious extension $K(|X|)$ $\rightarrow|K(X)|$ which is $K$-linear. Our main theorem will state that under certain circumstances, this map will be a weak equivalence of simplicial groups. Thus, $H_{*}(|X| ; K)=\pi_{*} K(|X|)=\pi_{*}|K(X)|$. There is a standard spectral sequence for $\pi_{*}|K(X)|$, for which $E_{p, q}^{1}=H_{p}(X[q] ; K)$.

In order to see that this spectral sequence includes the usual EilenbergMoore spectral sequence, suppose that we have a pair of maps $A \rightarrow C \leftarrow B$ of Kan complexes. Let $X[0]=A \times B$, and let $X[n]=X[0] \times C^{n}$ (in Rector's [4] terms, $X$ is the geometric cobar construction $\left.\mathscr{B}^{C}(A, B)\right)$. The coface maps are given by diagonals and by the maps $A \rightarrow C \leftarrow B$, codegeneracies by projections. Then if $D=|X|, D$ is easily seen to be the homotopy fiber product of $A$ and $B$ over $C$. Since $E_{p, *}^{1}=H_{*}(A) \otimes H_{*}\left(C^{p}\right)$ $\otimes H_{*}(B)=\mathscr{B}_{p}^{H_{*}(C)}\left(H_{*}(A), H_{*}(B)\right)$, the cobar construction on $H_{*}(A)$ and $H_{*}(B)$ over $H_{*}(C)$, we see that $E_{p, q}^{2}=\operatorname{Cotor}_{p, q}^{H_{*}^{*}(C)}\left(H_{*}(A), H_{*}(B)\right)$ (all homology groups with $K$-coefficients).

A second application of this spectral sequence is the computation of the homology of function spaces $Y^{Z}$. In order for our convergence conditions to hold, we need that $Y$ be a Kan complex, and that the connectivity of $Y$

AMS 1970 subject classifications. Primary 55H20. 
be as great as the dimension of $Z$. Let $X[m](n)=(Y(n))^{Z(m)}$ be the complex of set maps of $Z$ to $Y$. Then $|X|=Y^{Z}$, the simplicial function complex. Notice that $E_{*, q}^{1}=H_{*}\left(Y^{Z(q)}\right)=\otimes\left(H_{*}(Y)_{\sigma}: \sigma \in Z(q)\right)$, where $H_{*}(Y)_{\sigma}$ is a copy of $H_{*}(Y)_{\sigma}$ indexed by $\sigma$. Notice that for $0<p<2(\operatorname{conn}(Y)+1)$, $E_{p, q}^{1}=C^{q}\left(Z ; H_{p}(Y)\right)$, so that $E_{p, q}^{2}=H^{q}\left(Z ; H_{p}(Y)\right)$, at least if each $Z(q)$ is finite. Also, $E_{0, q}^{2}=0$ if $q \neq 0$, so that $E_{p, q}^{2}$ is determined for $p$ $<2(\operatorname{conn}(Y)+1)$. It is not hard to see that none of these groups contains any boundaries by comparing $Y$ with suitable Eilenberg-Mac Lane spaces. By the use of a vanishing theorem due to J. Kettner [2] for $E^{2}$, one can show that for $0 \leqq k \leqq 2(\operatorname{conn}(Y)-\operatorname{dim}(Z))+1, \quad H_{k}\left(Y^{Z}\right)$ $=\sum H^{i}\left(Z ; H_{k-i}(Y)\right)$. When $Y$ and $Z$ are basepointed, and we take $Y^{Z}$ to be the function complex of basepointed complexes, the same theorem holds with reduced homology groups for $Y, Z$ and $Y^{Z}$. This generalizes various known results (see for example Moore [3]). This result could also be obtained via Spanier duality.

When $Z$ is a sphere, the $E^{2}$-term is often computable. For $K=Z / 2 Z$, $Y$ a sphere of dimension greater than that of $Z, E^{2}=E^{\infty}$.

The generality of this spectral sequence allows one to define products, Steenrod operations, and Dyer-Lashoff operations on the spectral sequence (see Kettner [2]) for an account). Also, using Rector's techniques, one could replace $K$ by an arbitrary chain theory-or, indeed, produce the spectral sequence for a homology theory without any reference to how the homology theory was defined.

To finish, we give some conditions on $X$ for which we can show that $K(|X|) \rightarrow|K(X)|$ is a weak equivalence.

A simplicial set breaks up, in each degree, as the disjoint union of a degenerate part and an indecomposable part, and the indecomposable parts completely determine the degenerate parts. We shall require such a decomposition (or, rather its dual) for $X$. We shall call a cosimplicial space $X$ primitive if there are spaces and maps $p_{n}: X[n] \rightarrow P_{n}$, such that if $\alpha$ runs over the $\left(\begin{array}{l}n \\ k\end{array}\right)$ surjections $\alpha: \Delta_{n} \rightarrow \Delta_{k}$ for $k<n$, the product of $p_{n}$ and the $p_{k} X(\alpha)$ gives us an isomorphism

$$
X[n] \rightarrow P_{0}^{\left(\begin{array}{l}
n \\
0
\end{array}\right)} \times P_{1}^{\left(\begin{array}{l}
n \\
1
\end{array}\right)} \times \cdots \times P_{n-1}^{\left(\begin{array}{c}
n \\
n-1
\end{array}\right)} \times P_{n}^{\left(\begin{array}{c}
n \\
n
\end{array}\right)} .
$$

We call a primitive cosimplicial space $X$ convergent if every $X[n]$ is a Kan complex, each $P_{n}$ is $n$-connected, and if only finitely many $P_{n}$ are not contractable.

THEOREM 1. If $X$ is convergent, $K(|X|) \rightarrow|K(X)|$ is a weak equivalence.

Kettner has shown [2] that if $X$ is convergent, then the filtration of the spectral sequence is finite in each total degree, so the spectral sequence converges in the strong sense. 
It would seem that if one suitably modifies the geometric realization functor, one could prove Theorem 1 under the following definition of a convergent cosimplicial space. For a cosimplicial group $G_{*}$, let $N\left(G_{n}\right)$ be the intersection over all $\alpha: \Delta_{n} \rightarrow \Delta_{k}$ for $k<n$ of the kernels of $G(\alpha): G(n)$ $\rightarrow G(k)$. Call a cosimplicial space $X$ convergent if there is an unbounded nonnegative nondecreasing function $\varphi$ such that $N\left(\pi_{i}(X[n])\right)=0$ for $i \leqq n+\varphi(n)$.

If this second definition of convergence is sufficient to make Theorem 1 hold, I do not know, but there is reason to believe that it might be.

\section{BIBLIOGRAPHY}

1. A. K. Bousfield and D. M. Kan, Homotopy with respect to a ring, Proc. Sympos. Pure Math., vol. 22, Amer. Math. Soc., Providence, R.I., 1971, pp. 59-64.

2. J. Kettner, Thesis, M.I.T., Cambridge, Mass., 1972.

3. J. C. Moore, On a theorem of Borsuk, Fund. Math. 43 (1956), 195-201. MR 18, 662.

4. D. L. Rector, Steenrod operations in the Eilenberg-Moore spectral sequence, Comment Math. Helv. 45 (1970), 540-552.

Department of Mathematics, University of California at San Diego, la Jolla, California 92037 